\section{Ubiquitin ligase MKRN1 modulates telomere length homeostasis through a proteolysis of hTERT}

\author{
Jun Hyun Kim, ${ }^{1,2}$ Sun-Mi Park, ${ }^{1}$ Mi Ran Kang, ${ }^{1,2}$ \\ Sue-Young Oh, ${ }^{1,2}$ Tae H. Lee, ${ }^{1}$ Mark T. Muller, ${ }^{3}$ \\ and In Kwon Chung ${ }^{1,2,4}$ \\ ${ }^{1}$ Department of Biology and ${ }^{2}$ Molecular Aging Research \\ Center, Yonsei University, Seoul 120-749, Korea; \\ ${ }^{3}$ Department of Molecular Biology and Microbiology, \\ University of Central Florida, Orlando, Florida, 32826, USA
}

Telomere homeostasis is regulated by telomerase and a collection of associated proteins. Telomerase is, in turn, regulated by post-translational modifications of the ratelimiting catalytic subunit hTERT. Here we show that disruption of Hsp90 by geldanamycin promotes efficient ubiquitination and proteasome-mediated degradation of hTERT. Furthermore, we have used the yeast two-hybrid method to identify a novel RING finger gene (MKRN1) encoding an E3 ligase that mediates ubiquitination of hTERT. Overexpression of MKRN1 in telomerase-positive cells promotes the degradation of hTERT and decreases telomerase activity and subsequently telomere length. Our data suggest that MKRN1 plays an important role in modulating telomere length homeostasis through a dynamic balance involving hTERT protein stability.

Received December 13, 2004; revised version accepted February 15, 2005.

Telomeres are essential and functional components of the physical ends of eukaryotic chromosomes (Greider 1996). Properly capped telomeres enable cells to distinguish chromosome ends from double-strand breaks in the genome. Without functional telomeres, chromosomes are prone to nucleolytic degradation and end-toend fusion leading to cell death or possibly genetic recombination and survivor selection (Blasco et al. 1997). Most normal human somatic cells show a progressive loss of telomeric DNA during successive rounds of cell division due to a DNA end replication problem (Lingner et al. 1995). Thus, telomere shortening has been proposed as a ticketing mechanism that controls the replicative capacity of cells and cellular senescence (Harley 1991). Cells with extended replicative life spans have mechanisms to counteract loss of telomeric DNA. In most human cancer cells, telomere shortening is alleviated by telomerase, a ribonucleoprotein enzyme that is

[Keywords: Telomere; hTERT; Hsp90; ubiquitin; proteolysis] ${ }^{4}$ Corresponding author.

E-MAIL topoviro@yonsei.ac.kr; FAX 822-364-8660.

Article and publication are at http://www.genesdev.org/cgi/doi/10.1101/ gad. 1289405 composed of a catalytic subunit, hTERT, and its template RNA, hTR (Blackburn 1992).

Telomerase activity correlates with hTERT expression, implicating this catalytic subunit as the rate-limiting component of the telomerase holoenzyme (Nakamura and Cech 1998). Although telomerase activity is regulated by hTERT gene expression (Wang et al. 1998), several lines of evidence have suggested a post-translational regulation of telomerase activity. Post-translational modifications of telomerase may involve the interaction of hTERT with accessory proteins, chaperones, and polypeptide modifiers (Zhou and Lu 2001; G.E. Lee et al. 2004). For example, the molecular chaperone Hsp90 binds specifically to hTERT to promote the assembly of active telomerase both in a cell-free system and in intact cells (Holt et al. 1999). Moreover, inhibiting Hsp90 blocks the assembly of active telomerase, although the underlying mechanism is not fully elucidated.

In this present study, we report that disruption of Hsp90 function by geldanamycin (GA) promotes ubiquitination and proteasome-mediated degradation of hTERT. Furthermore, we have identified a novel hTERT-binding protein, Makorin RING finger protein 1 (encoded by the MKRN1 gene, a member of an ancient gene family) that functions as an E3 ligase and mediates ubiquitination of hTERT (Gray et al. 2000). Overexpression of MKRN1 in telomerase-positive cells decreases telomerase activity and telomere length. These observations suggest that MKRN-1, a post-translational modifier of hTERT, plays a negative role in telomere length homeostasis. These studies also define for the first time a physiological function associated with the very well-conserved Makorin gene family.

\section{Results and Discussion}

\section{hTERT is sensitive to the Hsp90 antagonist geldanamycin}

Inhibition of Hsp90 function by GA prevents the assembly of active telomerase (Holt et al. 1999). Because GA mediates the dissociation of Hsp90 from its client proteins and promotes rapid degradation by the proteasome (Goetz et al. 2003), we predicted that hTERT would be sensitive to the Hsp90 antagonist. To investigate this possibility, we transfected H1299 cells with Flag-tagged hTERT and assessed protein levels in the absence or presence of GA. hTERT protein was highly expressed in untreated cells but was down-regulated in a time-dependent manner in response to GA treatment, with $>50 \%$ of the protein being eliminated within $2 \mathrm{~h}$ of treatment (Fig. 1A, lanes 1-5 in upper blot). TRF1 expression levels were not affected by GA treatment (Fig. 1A, middle blot). GA also decreased the level of hTERT expression in a dose-dependent manner (Fig. 1B). To verify the ability of GA to down-regulate endogenous hTERT levels, H1299 cells were treated with GA and immunoblotted using a polyclonal anti-hTERT antibody. Treatment of cells with GA significantly decreased endogenous hTERT levels (Fig. 1C, upper blot).

Because the Hsp90 chaperone complex binds to hTERT and influences proper assembly with hTR to form an active enzyme (Holt et al. 1999; Forsythe et al. 

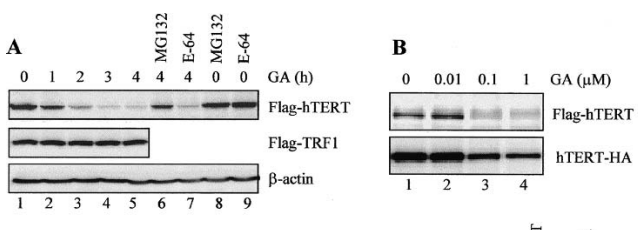

C
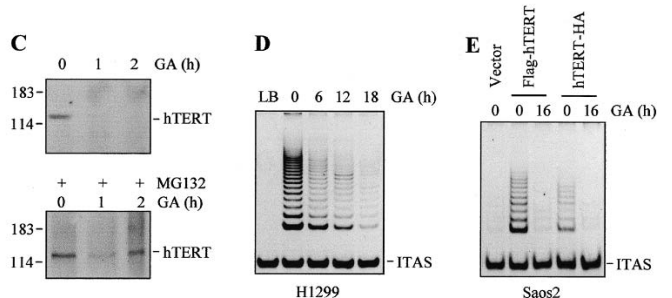

Figure 1. hTERT is sensitive to the Hsp90 antagonist geldanamycin. (A) Down-regulation of ectopically expressed hTERT by GA. H1299 cells transfected with Flag-hTERT or Flag-TRF1 were treated with $0.1 \mu \mathrm{M}$ GA for the indicated times. Lysates were resolved on $8 \%$ SDS-PAGE and analyzed by immunoblotting using an anti-Flag antibody probe. Cells were pretreated with $10 \mu \mathrm{M}$ MG132 or E-64 for $2 \mathrm{~h}$, either alone or before treatment with GA. (B) Concentration dependence of GA on hTERT. H1299 cells transfected with FlaghTERT or hTERT-HA were treated for $2 \mathrm{~h}$ with the GA concentration indicated. The hTERT proteins were visualized with anti-Flag or anti-HA antibodies as marked by each blot. (C) Down-regulation of endogenous hTERT by GA. H1299 cells were treated with $0.1 \mu \mathrm{M}$ GA for the indicated times, and cell lysates were analyzed by immunoprecipitation with anti-hTERT antibody followed by immunoblotting using the same antibody probe. Cells were pretreated with $10 \mu \mathrm{M}$ MG132 for $2 \mathrm{~h}$, either alone or before treatment with GA. Molecular weight markers are shown in kilodaltons. (D) H1299 cells were treated with $0.1 \mu \mathrm{M}$ GA for the indicated times, and cell lysates were analyzed for telomerase activity by the TRAP assay. The lane labeled LB corresponds to the negative control (lysis buffer only). ITAS represents the internal telomerase assay standard. $(E)$ Saos- 2 cells transfected with Flag-hTERT or hTERT-HA or empty vector were untreated or treated with $0.1 \mu \mathrm{M}$ GA for $16 \mathrm{~h}$, and cell lysates were analyzed for telomerase activity.

2001), we tested the influence of GA-induced degradation of hTERT on telomerase activity in H1299 cells. Incubation with GA reduced telomerase activity in a time-dependent manner (Fig. 1D). To more clearly determine the effects of GA on telomerase activity, N-terminal Flag-tagged hTERT and C-terminal HA-tagged hTERT constructs were transfected to Saos- 2 cells (these cells lack detectable telomerase activity) (Kim et al. 2003). The forced expression of exogenous hTERT produced detectable telomerase activity in these cells (Fig. 1E). Furthermore, incubation with GA reduced telomerase activity to near basal levels. These results suggest that Hsp90 is not only important for the proper conformation of hTERT but also for maintaining telomerase assembly.

\section{hTERT is ubiquitinated and degraded via the proteasomal pathway}

Reduction in hTERT levels could be due to degradation and/or turnover of the protein. To examine whether the GA-mediated degradation of hTERT proceeds via the proteasome, we pretreated $\mathrm{H} 1299$ cells for $2 \mathrm{~h}$ with the specific proteasome inhibitor MG132, and subsequently incubated with GA for $4 \mathrm{~h}$. Incubation of cells with MG132 rescued the GA-induced down-regulation of hTERT (Fig. 1A, lane 6 in upper blot), whereas pretreatment of cells with the lysosomal proteolysis inhibitor
E-64 had no effect on hTERT levels (Fig. 1A, cf. lanes 6 and 7). In the absence of GA treatment, incubation of cells with either MG132 or E-64 alone did not cause a significant change in the levels of hTERT expression relative to untreated controls (Fig. 1A, lanes 8,9 in upper blot). These results indicate that GA-induced downregulation of hTERT is due to proteasome-mediated degradation. Note that endogenous hTERT levels were similarly affected. Specifically, pretreating cells with MG132 followed by GA, reversed the degradation of endogenous hTERT protein (Fig. 1C, cf. upper and lower blots).

GA-mediated protein degradation by the proteasome depends on conjugation of ubiquitin to the Hsp90 client protein (Isaacs et al. 2002). To determine whether hTERT is ubiquitinated in vivo prior to its degradation, H1299 cells cotransfected with Flag-tagged hTERT and HA-tagged ubiquitin constructs were treated with GA, ALLnN (a general proteasome inhibitor), or both. The immunoprecipitated products were evaluated by immunoblotting with selected probes as described in the legend for Figure 2. Treatment of GA alone decreased hTERT levels and GA plus ALLnN reversed this trend (Fig. 2A, cf. lanes 3 and 4 in upper blot). Treatment with ALLnN alone did not affect hTERT levels relative to controls (Fig. 2A, cf. lanes 1 and 2 in upper blot). Note that the amount of high-molecular-weight smearing was much greater in the ALLnN-treated cells (Fig. 2A, cf. lanes 1,3 and 2,4 in upper blot). To confirm that these slower migrating bands represented ubiquitinated hTERT, anti-Flag immunoprecipitates were probed with anti-HA antibody to illuminate ubiquitin-modified hTERT (Fig. 2A, lower blot). Again, ubiquitinated hTERT was dramatically elevated in the presence of the proteasome inhibitor ALLnN (Fig. 2A, cf. lanes 1,3 and 2,4 in lower blot). These high-molecular-weight smears are diagnostic for polyubiquitinated proteins (Girnita et al. 2003). This modification was substantially enhanced by the treating cells with both ALLnN and GA (Fig. 2A, cf. lanes 2 and 4 in lower blot). In addition, hTERT displayed enhanced GA-induced ubiquitination in a dosedependent manner in the presence of MG132 (Fig. 2B).
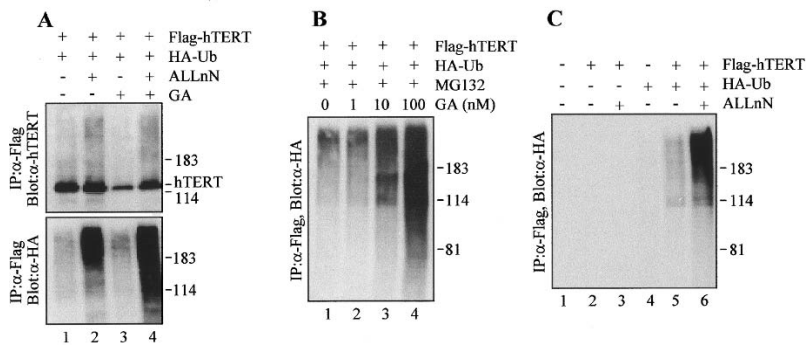

Figure 2. hTERT is ubiquitinated prior to proteasome-mediated degradation. (A) H1299 cells were cotransfected with Flag-hTERT and HA-ubiquitin. Cells were either untreated or treated for $2 \mathrm{~h}$ with $10 \mu \mathrm{g} / \mathrm{mL}$ ALLnN or $0.1 \mu \mathrm{M}$ GA, or a combination of the two as specified. Immunoprecipitation was performed with anti-Flag antibody before probing with anti-hTERT or anti-HA antibodies as indicated. Molecular weight markers are shown in kilodaltons. $(B)$ H1299 cells cotransfected with Flag-hTERT and HA-ubiquitin were treated for $1 \mathrm{~h}$ with the GA concentration indicated in the presence of $10 \mu \mathrm{M}$ MG132. (C) H1299 cells cotransfected with Flag-hTERT and HA-ubiquitin were either untreated or treated for $2 \mathrm{~h}$ with 10 $\mu \mathrm{g} / \mathrm{mL}$ ALLnN as specified. Proteins were immunoprecipitated with anti-Flag antibody followed by immunoblotting using an anti-HA antibody probe. 
These data indicate that hTERT is ubiquitinated in GAtreated cells prior to proteasome-dependent degradation in a manner similar to other Hsp90 client proteins (Isaacs et al. 2002). On the basis of these results, we propose that two counteracting processes mediate hTERT levels: proteasomal degradation by the ubiquitination pathway and rescue (or protection) from degradation by Hsp90. The dynamic balance between these two processes determines equilibrium levels of hTERT, which then sets cellular telomerase activity. It is also worth noting that polyubiquitinated hTERT forms were significantly elevated by ALLnN in the absence of GA treatment (Fig. 2C, lanes 5,6). These results suggest that hTERT is subject to ubiquitination even in the absence of GA.

\section{Identification of a RING finger protein that interacts with hTERT}

Ubiquitination is carried out by a cascade of reactions catalyzed by ubiquitin-activating enzyme (E1), ubiquitin-conjugating enzyme (E2), and ubiquitin ligase (E3) (Pickart 2001). E3 proteins are known to play a pivotal role in determining the specificity of the system by recognizing target substrates. We therefore established a yeast two-hybrid system to search for an E3 ligase involved in hTERT degradation. With the C-terminal domain of hTERT as bait (residues 946-1132), we identified proteins that interact with hTERT (Fig. 3A). One of the isolated clones contains a $\mathrm{C}_{3} \mathrm{HC}_{4}$-type $\mathrm{RING}$ finger domain that is present in many E3 ubiquitin ligases (Joazeiro et al. 1999). We discovered that the protein was identical to Makorin RING finger protein 1 (MKRN1) (Gray et al. 2000). MKRN1 is uniformly expressed in all tissues and encodes a putative ribonucleoprotein with a distinctive array of zinc-finger motifs, including four $\mathrm{C}_{3} \mathrm{H}$ zinc-finger motifs, an unusual Cys-His motif, and a highly conserved RING finger domain (Fig. 3B). MKRN1 orthologs have been identified in a wide spectrum of species (from invertebrates to vertebrates), suggesting an ancient origin of this highly conserved gene (Gray et al. 2000).

The physical interaction between MKRN1 and hTERT was examined by coimmunoprecipitation experiments in cells cotransfected with C-terminal HA-tagged hTERT and MKRN1-V5 expression vectors (Fig. 3C). The data show that MKRN1 was barely detectable in lysates directly probed with anti-V5 antibody (Fig. 3C, lanes 1,3). This suggests that MKRN1 may be rapidly degraded through auto-ubiquitination like other RING finger proteins that promote their own ubiquitination/degradation (Joazeiro and Weissman 2000). In the presence of ALLnN, however, there was a significant increase in both unmodified and ubiquitinated MKRN1 species (Fig. 3C, lanes 2,4). Immunoprecipitation of cell lysates with anti-HA antibody (hTERT) and subsequent immunoblot analysis with anti-V5 antibody (MKRN1) revealed that hTERT coimmunoprecipitated with MKRN1 in cells treated with ALLnN but not in negative drug control cells (Fig. 3C, cf. lanes 7 and 8), suggesting that hTERT binds to MKRN1 in mammalian cells. When this experiment was modified using N-terminal Flag-tagged hTERT and MKRN1-V5 expression vectors with a different proteasome inhibitor (MG132), essentially identical results were obtained (Fig. 3D).
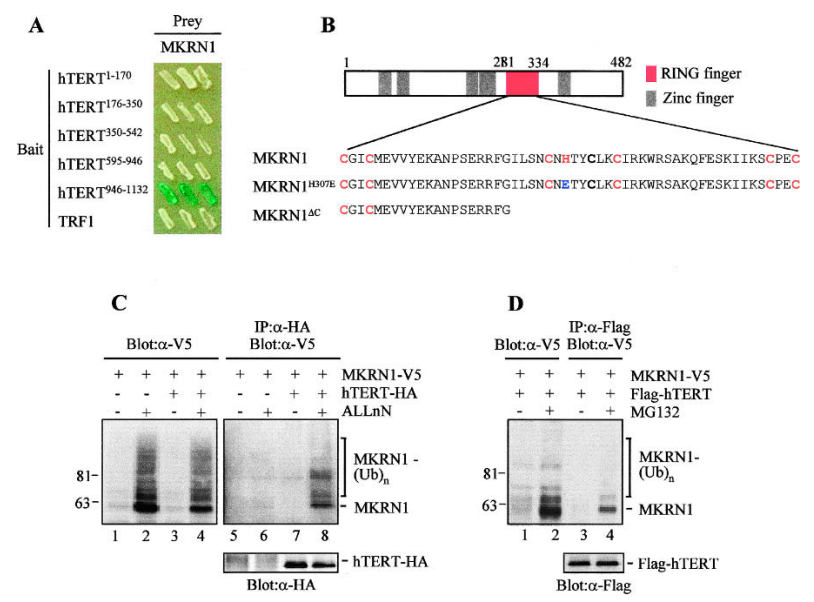

Figure 3. MKRN1 interacts with hTERT. (A) Analysis of the physical interaction between hTERT and MKRN1 using the yeast twohybrid assay. Five domains of hTERT were analyzed for binding to MKRN1. TRF1 bait was used as a negative control. Blue signal on the SG-HWU/X plate indicates activation of the reporter gene LacZ. (S) Synthetic; (G) galactose; (H) histidine $(-)$; (W) tryptophan $(-)$; (U) uracil (-); (X) X-gal. (B) Schematic diagram of MKRN1 domains. Amino acid sequences surrounding the RING finger domain in MKRN1 and mutants used in this study. Cysteines and a histidine in the RING finger domain are shown in red. The mutated residue in MKRN1 ${ }^{\mathrm{H} 307 \mathrm{E}}$ is shown in blue. (C) H1299 cells cotransfected with hTERT-HA and MKRN1-V5 were either untreated or treated for $2 \mathrm{~h}$ with $10 \mu \mathrm{g} / \mathrm{mL}$ ALLnN. Anti-HA immunoprecipitates were analyzed by immunoblotting using an anti-V5 antibody probe. The left panel shows the immunoblot of cell lysates $(3 \%$ of the precipitated lysates) probed with anti-V5 antibody. hTERT-HA was visualized with an anti-HA antibody probe. (D) H1299 cells cotransfected with Flag-hTERT and MKRN1-V5 were either untreated or treated for $2 \mathrm{~h}$ with $10 \mu \mathrm{M}$ MG132. Anti-Flag immunoprecipitates were analyzed by immunoblotting using an anti-V5 antibody probe. The left panel shows the immunoblot of cell lysates $3 \%$ of the precipitated lysates) probed with anti-V5 antibody. Flag-hTERT was visualized with an anti-Flag antibody probe.

\section{MKRN1 is an E3 ubiquitin-ligase that enhances ubiquitination of hTERT}

We next investigated the involvement of MKRN1 in ubiquitination of hTERT. In vitro ubiquitination assays using purified components showed that the C-terminal domain (residues 946-1132) of hTERT was efficiently ubiquitinated by wild-type MKRN1, but not by mutants MKRN1 $1^{\mathrm{H} 307 \mathrm{E}}$ or MKRN1 ${ }^{\Delta \mathrm{C}}$ (Fig. 4A). Thus, while MKRN1 is an E3 ligase for hTERT, mutating the RING finger domain destroys this activity in vitro, indicating that the RING finger domain is indispensable for E3 activity.

To investigate whether MKRN1 contributes to the ubiquitination of hTERT in vivo, H1299 cells were cotransfected with Flag-hTERT, HA-ubiquitin, and MKRN1-V5 constructs, and immunoprecipitated with anti-Flag antibody followed by immunoblotting with anti-HA antibody. Ubiquitin conjugates of hTERT were easily detected in the presence of MG132 (Fig. 4B, lane 2 in upper blot). The amount of ubiquitinated hTERT was significantly reduced in cells cotransfected with MKRN1 plus MKRN1 ${ }^{\mathrm{H} 307 \mathrm{E}}$ (Fig. 4B, lane 6) compared to cells transfected with MKRN1 alone (Fig. 4B, lane 2) or in cells cotransfected with MKRN1 plus MKRN1 ${ }^{\Delta C}$ (Fig. $4 \mathrm{~B}$, lane 4). These data demonstrate that mutating the RING finger in MKRN1 ${ }^{\mathrm{H} 307 \mathrm{E}}$ does not impair its ability 

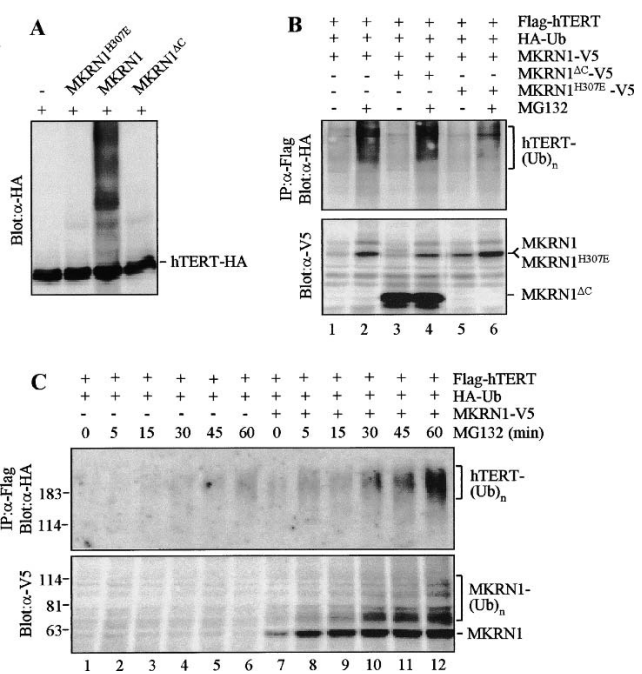

Figure 4. MKRN1 functions as an E3 ubiquitin-ligase for hTERT in vitro and in vivo. (A) HA-tagged hTERT (residues 946-1132) was incubated with MKRN1 or mutants in the presence of E1, E2, and His $_{6}$-ubiquitin. Samples were resolved by $6 \%$ SDS-PAGE and analyzed by immunoblotting with an anti-HA antibody probe. $(B)$ H1299 cells were cotransfected with Flag-hTERT, HA-ubiquitin, MKRN1-V5, and together with either MKRN1 ${ }^{\mathrm{H} 307 \mathrm{E}}-\mathrm{V} 5$ or MKRN1 ${ }^{\Delta \mathrm{C}}-\mathrm{V} 5$ as specified. Cells were untreated or treated with 10 $\mu \mathrm{M}$ MG132 for $2 \mathrm{~h}$. Anti-Flag immunoprecipitates and cell lysates were analyzed by immunoblotting with anti-HA antibody and antiV5 antibody probes, respectively. (C) H1299 cells cotransfected with Flag-hTERT and HA-ubiquitin, and together with or without MKRN1-V5 as indicated, were treated with $10 \mu \mathrm{M}$ MG132 for the indicated times. Anti-Flag immunoprecipitates and cell lysates were analyzed by immunoblotting with anti-HA antibody and anti-V5 antibody probes, respectively.

to interact with hTERT but disrupts the domain structure of the ubiquitin ligase activity, thereby yielding a dominant-negative phenotype. When the levels of ectopically expressed MKRN1 and mutants were examined, MKRN1 expression was clearly elevated by MG132 treatment (Fig. 4B, cf. lanes 1 and 2 in lower blot). In contrast, MG132 had no effect on the amount of MKRN1 $^{\triangle C}$ (Fig. 4B, cf. lanes 3 and 4), suggesting that the deleted C-terminal region is required for auto-ubiquitination of MKRN1. When both MKRN1 and MKRN1 ${ }^{\mathrm{H} 307 \mathrm{E}}$ were cotransfected, we were able to detect MKRN1 ${ }^{\mathrm{H} 307 \mathrm{E}}$ in the absence of MG132 although the expression level was slightly less (approximately twofold) compared with that elicited by the MG132 treatment (Fig. 4B, cf. lanes 5 and 6). Thus, mutating the histidine residue in the RING finger motif inhibits auto-ubiquitination.

To examine the in vivo role of MKRN1 in ubiquitination of hTERT, we cotransfected H1299 cells with FlaghTERT plus HA-ubiquitin and immunoprecipitated with anti-Flag antibody followed by immunoblotting with anti-HA antibody. Although no hTERT-ubiquitin conjugates were observed in untreated cells (Fig. 4C, lane 1 in upper blot), low levels were detected after 45-60 min of MG132 treatment (Fig. 4C, lanes 5,6). In marked contrast, coexpression of MKRN1 caused a clear and dramatic increase in the amount of ubiquitinated hTERT species (Fig. 4C, lanes 7-12). When the expression levels of MKRN1 were examined by immunoblotting with anti-V5 antibody, both unmodified and polyubiquitinated MKRN1 species increased in a time-dependent manner in response to MG132 treatment (Fig. 4C, lanes
7-12 in lower blot). On the basis of these data, we conclude that MKRN1 is an E3 ligase for hTERT ubiquitination in mammalian cells. Although our findings show that MKRN1 associates with and efficiently ubiquitinates hTERT, our data do not exclude the existence of other E3 ligases that target hTERT. The Hsp90/Hsp70associated U-box ubiquitin ligase CHIP /C terminus Hsc70-interacting protein) may mediate the GA-stimulated degradation of some Hsp90 client proteins (Connell et al. 2001). Whether CHIP E3 activity regulates in vivo stability of hTERT remains to be elucidated. We are presently investigating the roles of CHIP and other E3 ligases in the GA-induced as well as the constitutive ubiquitination of hTERT.

\section{Overexpression of MKRN1 decreased telomerase activity and telomere length}

Because MKRN1 functions as an E3 ligase for hTERT ubiquitination in vivo, we examined whether ectopic expression of MKRN1 affects telomerase activity. We established HT1080 cell lines stably expressing MKRN1 (or an empty vector negative control). Levels of ectopically expressed MKRN1 were examined by immunoblotting with anti-V5 antibody (Fig. 5A). Treatment of MG132 resulted in a substantial increase in MKRN1 compared to untreated cells (Fig. 5A, cf. lanes 3 and 6). Cells expressing MKRN1 and the empty vector grew normally and exhibited no detectable differences in growth

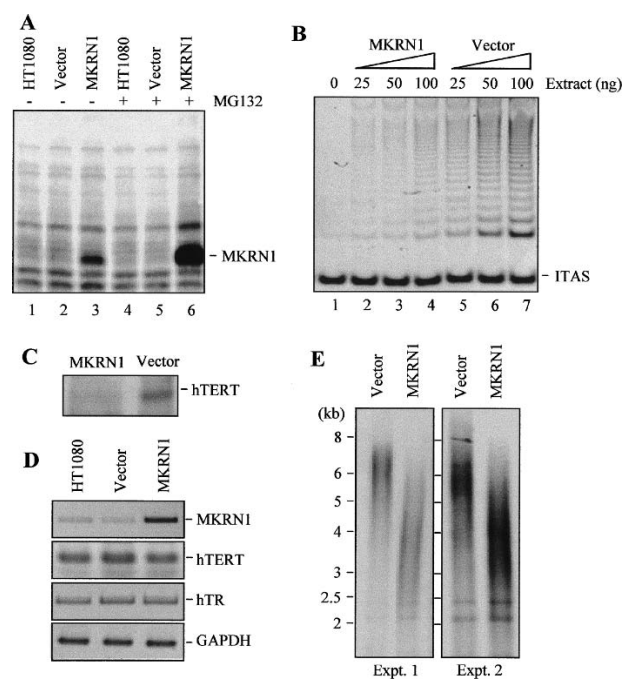

Figure 5. Overexpression of MKRN1 decreases telomerase activity and telomere length. $(A)$ HT1080 cells were stably transfected with MKRN1-V5 or an empty vector. The cells were untreated or treated for $2 \mathrm{~h}$ with $10 \mu \mathrm{M}$ MG132. Cell lysates were analyzed by immunoblotting with anti-V5 antibody probe. (B) Stable HT1080 cell lines (MKRN1 and vector) were harvested at $45 \mathrm{PD}$, and telomerase activities were measured with different amounts of proteins using the TRAP assay. (C) Cell lysates from stable HT1080 cell lines were analyzed by immunoprecipitation with anti-hTERT antibody followed by immunoblotting using the same antibody probe. $(D)$ Representative results of RT-PCR analysis for the expression of MKRN1, hTERT, and hTR in stable HT1080 cell lines. RT-PCR products from each sample were normalized to glyceraldehyde-3phosphate dehydrogenase (GAPDH) signal. (E) Stable HT1080 cell lines were harvested at $45 \mathrm{PD}$, and genomic DNA was digested with RsaI and HinfI, followed by Southern blotting using a telomere repeat probe. 
rates or morphology over 45 population doublings (PD). At 45 PD, we compared telomerase activity in stable cell lines expressing MKRN1 and the empty vector. Telomerase activity in cells expressing MKRN1 was significantly decreased relative to the control cell line (Fig. 5B). The overexpression of MKRN1 resulted in a down-regulation of the endogenous hTERT protein (Fig. 5C). These results suggest that MKRN1 acts as a negative regulator of telomerase. However, it is not clear whether this decrease is due to a MKRN1-related decrease in the expression of $h T E R T$ or $h T R$ genes. To address this possibility, the impact of MKRN1 on gene expression of $h T E R T$ and $h T R$ was evaluated using RT-PCR analysis (J.H. Kim et al. 2002). Whereas the MKRN1 mRNA level was much higher in cells expressing the gene /relative to control cells), we observed no significant differences in steadystate levels of hTERT mRNA or hTR transcripts in cell lines expressing MKRN1 and the empty vector (Fig. 5D). To examine whether the MKRN1-mediated decrease in telomerase activity was attended by changes in telomere lengths, we performed a terminal restriction fragment (TRF) size analysis (G.E. Lee et al. 2004). Control cells exhibited an average telomere length of $\sim 6 \mathrm{~kb}$ with most fragments spread over a range of $\sim 5$ to $7 \mathrm{~kb}$ (Fig. 5E). Telomeres were clearly shortened in the clones expressing MKRN1 with a decrease in TRF length to $\sim 4 \mathrm{~kb}$ and spanning down to roughly $2.5 \mathrm{~kb}$. These findings suggest that MKRN1 exerts a negative role in telomere length maintenance and/or regulation.

The data presented here demonstrate that MKRN1 markedly enhances hTERT ubiquitination in the absence of GA treatment. This suggests that MKRN1 functions as an E3 ligase for the constitutive ubiquitination of mature hTERT assembled with hTR in the nucleus when the molecular chaperone Hsp90 is intact. Additionally, MKRN1 may perform this same function with newly synthesized hTERT in cytoplasm. Ubiquitination of hTERT could be regulated by changes in the expression and activity of MKRN1 and de-ubiquitinating enzymes. Moreover, continued expression of MKRN1 in telomerase-positive cells resulted in a decrease in telomerase activity and cells with shortened telomeres. Thus, MKRN1 regulates telomerase activity and telomere length through dynamic control of hTERT protein stability. The function of telomerase during development, aging, and cancer has been extensively studied (S.H. Kim et al. 2002). MKRN1, a post-translational modifier of hTERT, may play an important role in telomere length maintenance and/or regulation through ubiquitin-mediated proteolysis of hTERT.

\section{Materials and methods}

\section{Cell lines and culture conditions}

The human lung carcinoma cell line H1299 was cultured in RPMI-1640 medium, and the human fibrosarcoma cell line HT1080 was cultured in Dulbecco's modified Eagle's medium supplemented with $10 \%$ fetal bovine serum, 100 units $/ \mathrm{mL}$ penicillin, and $100 \mu \mathrm{g} / \mathrm{mL}$ streptomycin in $5 \%$ $\mathrm{CO}_{2}$ at $37^{\circ} \mathrm{C}$. The human osteosarcoma cell line Saos-2 was maintained in McCoy's 5A medium containing $15 \%$ fetal bovine serum, 100 units/ $\mathrm{mL}$ penicillin, and $100 \mu \mathrm{g} / \mathrm{mL}$ streptomycin. To establish cell lines stably expressing MKRN1, the MKRN1-V5 construct was transfected into HT1080 cells using Lipofectamine Plus according to the manufacturer's instructions (Invitrogen). Multiple independent single clones were isolated and checked for protein expression by immunoblotting with antiV5 antibody.
Expression vectors

The Flag-hTERT expression vector was constructed by cloning the fulllength hTERT cDNA into a pCMV-Tag2 vector (Stratagene). The hTERTHA expression vector has been described (Seimiya et al. 2000). The HAubiquitin expression vector was constructed by cloning the BamHI and XbaI fragment encoding the ubiqutin cDNA into pcDNA3.1 (Invitrogen) (J.S. Lee et al. 2004). The MKRN1-V5 expression vector was constructed by inserting the EcoRI and NotI fragment from the full-length MKRN1 cDNA into pcDNA3.1/V5. The expression vector for GST-hTERT-HA was constructed by cloning the fragment encoding the $\mathrm{C}$-terminal region (amino acids 946-1132) of hTERT into pGEX-5X-2 (Amersham Biosciences). MKRN1 and its mutants were subcloned into pGEX-6P-1 for expression in bacteria.

Yeast two-hybrid screening

Yeast two-hybrid screening was performed as described (G.E. Lee et al. 2004). Briefly, the yeast strain EGY48 harboring pLexA-hTERT (amino acids 946-1132) and pSH18-34 was transformed by the lithium acetate method with a HeLa cDNA library fused to the activation domain vector pB42AD (Clontech).

Immunoprecipitation and immunoblotting

H1299 cells were transiently transfected with the expression vectors as indicated using TransFast transfection reagent (Promega). Cells were lysed with buffer containing $0.5 \%$ NP-40, 50 mM Tris-HCl (pH 7.5), 150 $\mathrm{mM} \mathrm{NaCl}$, and $2 \mathrm{mM}$ phenyl methylsulphonyl fluoride. After centrifugation at 12,000 rpm for $30 \mathrm{~min}$, the supernatants were immunoprecipitated for $2 \mathrm{~h}$ with anti-Flag (Sigma) or anti-HA (Santa Cruz biotechnology) antibodies and then incubated with Sepharose beads conjugated to Protein G (Amersham Biosciences). When ubiquitin conjugates of hTERT were detected, immunoprecipitation was performed with lysis buffer containing $0.1 \%$ SDS to dissociate its interacting proteins. Immunoblot analyses were performed using anti-hTERT (Santa Cruz biotechnology), anti-V5 (Invitrogen), anti-HA, and anti-Flag antibodies.

In vitro ubiquitination assay

GST-hTERT-HA expressed in bacteria was used as substrate for in vitro ubiquitination assay as previously described (Park et al. 2004). Purified wild-type or mutant MKRN1 as an E3 enzyme was added to the ubiquitination reaction as indicated. The reaction mixtures were incubated at $30^{\circ} \mathrm{C}$ for $60 \mathrm{~min}$ and terminated with sample loading buffer and run on SDS-PAGE. Slow migrating ubiquitin conjugates were visualized by immunoblot analysis with anti-HA antibody.

Telomerase assays

The telomeric repeat amplification protocol (TRAP) was used as previously described (Kim et al. 2003). Cell extracts (200 ng of protein) were added to telomerase extension reactions and incubated for $20 \mathrm{~min}$ at $37^{\circ} \mathrm{C}$. PCR was performed using the TS primer and ACX primer for 30 cycles (denaturation at $94^{\circ} \mathrm{C}$ for $30 \mathrm{sec}$, annealing at $60^{\circ} \mathrm{C}$ for $30 \mathrm{sec}$, and extension at $72^{\circ} \mathrm{C}$ for $30 \mathrm{sec}$. As an internal telomerase assay standard, NT and TSNT primers were added to the PCR mixture as previously described (Kim and Wu 1997). Telomerase products were resolved by electrophoresis on a $12 \%$ nondenaturing polyacrylamide gel. Bands were then visualized by staining with SYBR Green (Molecular Probes). The signal intensity was quantified with a LAS-1000 Plus Image analyzer (Fuji Photo Film).

\section{Terminal restriction fragment (TRF) analysis}

To measure the telomere length, genomic DNA was digested with RsaI and HinfI and separated on a $0.7 \%$ agarose gel. DNA samples were transferred to a nylon membrane (Hybond $\mathrm{N}^{+}$; Amersham Biosciences) and hybridized with a ${ }^{32} \mathrm{P}$-labeled probe (TTAGGG) ${ }_{20}$. Signals were detected by a phosphoimage analyzer (Fuji Photo Film).

\section{Acknowledgments}

We thank Jong-Bok Yoon for the HA-ubiquitin expression vector and Hiroyuki Seimiya for the hTERT-HA expression vector. This work was supported in part by grants from the Korean Ministry of Health and Welfare through the Molecular Aging Research Center and from the Korea Science and Engineering Foundation through the Protein Network 
Research Center (to I.K.C.), and a grant from the Korean Ministry of Science and Technology through 21C Frontier Project (to T.H.L.).

\section{References}

Blackburn, E.H. 1992. Telomerases. Annu. Rev. Biochem. 61: 113-129. Blasco, M.A., Lee, H.W., Hande, M.P., Samper, E., Lansdorp, P.M., DePinho, R.A., and Greider, C.W. 1997. Telomere shortening and tumor formation by mouse cells lacking telomerase RNA. Cell 91: 25-34.

Connell, P., Ballinger, C.A., Jiang, J., Wu, Y., Thompson, L.J., Hohfeld, J., and Patterson, C. 2001. The co-chaperone CHIP regulates protein triage decisions mediated by heat-shock proteins. Nat. Cell Biol. 3: 93-96.

Forsythe, H.L., Jarvis, J.L., Turner, J.W., Elmore, L.W., and Holt, S.E. 2001. Stable association of hsp90 and p23, but Not hsp70, with active human telomerase. J. Biol. Chem. 276: 15571-15574.

Girnita, L., Girnita, A., and Larsson, O. 2003. Mdm2-dependent ubiquitination and degradation of the insulin-like growth factor 1 receptor. Proc. Natl. Acad. Sci. 100: 8247-8252.

Goetz, M.P., Toft, D.O., Ames, M.M., and Erlichman, C. 2003. The Hsp90 chaperone complex as a novel target for cancer therapy. Ann. Oncol. 14: 1169-1176.

Gray, T.A., Hernandez, L., Carey, A.H., Schaldach, M.A., Smithwick, M.J., Rus, K., Marshall Graves, J.A., Stewart, C.L., and Nicholls, R.D. 2000. The ancient source of a distinct gene family encoding proteins featuring RING and $\mathrm{C}(3) \mathrm{H}$ zinc-finger motifs with abundant expression in developing brain and nervous system. Genomics 66: 76-86.

Greider, C.W. 1996. Telomere length regulation. Annu. Rev. Biochem. 65: 337-365.

Harley, C.B. 1991. Telomere loss: Mitotic clock or genetic time bomb? Mutat. Res. 256: 271-282

Holt, S.E., Aisner, D.L., Baur, J., Tesmer, V.M., Dy, M., Ouellette, M., Trager, J.B., Morin, G.B., Toft, D.O., Shay, J.W., et al. 1999. Functional requirement of $\mathrm{p} 23$ and $\mathrm{Hsp} 90$ in telomerase complexes. Genes \& Dev. 13: 817-826.

Isaacs, J.S., Jung, Y.J., Mimnaugh, E.G., Martinez, A., Cuttitta, F., and Neckers, L.M. 2002. Hsp90 regulates a von Hippel Lindau-independent hypoxia-inducible factor-1 $\alpha$-degradative pathway. I. Biol. Chem. 277: 29936-29944.

Joazeiro, C.A. and Weissman, A.M. 2000. RING finger proteins: Mediators of ubiquitin ligase activity. Cell 102: 549-552.

Joazeiro, C.A., Wing, S.S., Huang, H., Leverson, J.D., Hunter, T., and Liu, Y.C. 1999. The tyrosine kinase negative regulator c-Cbl as a RINGtype, E2-dependent ubiquitin-protein ligase. Science 286: 309-312.

Kim, N.W. and Wu, F. 1997. Advances in quantification and characterization of telomerase activity by the telomeric repeat amplification protocol (TRAP). Nucleic Acids Res. 25: 2595-2597.

Kim, J.H., Lee, G.E., Kim, J.C., Lee, J.H., and Chung, I.K. 2002. A novel telomere elongation in an adriamycin-resistant stomach cancer cell line with decreased telomerase activity. Mol. Cell 13: 228-236.

Kim, S.H., Kaminker, P., and Campisi, J. 2002. Telomeres, aging and cancer: In search of a happy ending. Oncogene 21: 503-511.

Kim, J.H., Kim, J.H., Lee, G.E., Lee, J.E., and Chung, I.K. 2003. Potent inhibition of human telomerase by nitrostyrene derivatives. Mol. Pharmacol. 63: 1117-11124.

Lee, G.E., Yu, E.Y., Cho, C.H., Lee, J., Muller, M.T., and Chung, I.K. 2004 DNA-protein kinase catalytic subunit-interacting protein KIP binds telomerase by interacting with human telomerase reverse transcriptase. J. Biol. Chem. 289: 34750-34755.

Lee, J.S., Hong, U.S., Lee, T.H., Yoon, S.K., and Yoon, J.B. 2004. Mass spectrometric analysis of tumor necrosis factor receptor-associated factor 1 ubiquitination mediated by cellular inhibitor of apoptosis 2 . Proteomics 4: 3376-3382.

Lingner, J., Cooper, J.P., and Cech, T.R. 1995. Telomerase and DNA end replication: No longer a lagging strand problem? Science 269: 15331534

Nakamura, T.M. and Cech, T.R. 1998. Reversing time: Origin of telomerase. Cell 92: 587-590.

Park, S.M., Yoon, J.B., and Lee, T.H. 2004. Receptor interacting protein is ubiquitinated by cellular inhibitor of apoptosis proteins (c-IAP1 and c-IAP2) in vitro. FEBS Lett. 566: 151-156.

Pickart, C.M. 2001. Mechanisms underlying ubiquitination. Annu. Rev. Biochem. 70: 503-533.
Seimiya, H., Sawada, H., Muramatsu, Y., Shimizu, M., Ohko, K., Yamane, K., and Tsuruo, T. 2000. Involvement of 14-3-3 proteins in nuclear localization of telomerase. EMBO J. 19: 2652-2661.

Wang, J., Xie, L.Y., Allan, S., Beach, D., and Hannon, G.J. 1998. Myc activates telomerase. Genes \& Dev. 12: 1769-1774.

Zhou, X.Z. and Lu, K.P. 2001. The Pin2/TRF1-interacting protein PinX1 is a potent telomerase inhibitor. Cell 107: 347-359. 


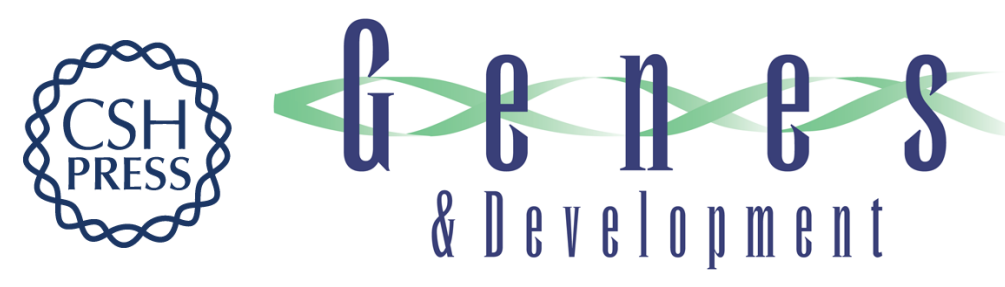

\section{Ubiquitin ligase MKRN1 modulates telomere length homeostasis through a proteolysis of hTERT}

Jun Hyun Kim, Sun-Mi Park, Mi Ran Kang, et al.

Genes Dev. 2005, 19:

Access the most recent version at doi:10.1101/gad.1289405

References This article cites 25 articles, 9 of which can be accessed free at: http://genesdev.cshlp.org/content/19/7/776.full.html\#ref-list-1

License

Email Alerting

Receive free email alerts when new articles cite this article - sign up in the box at the top Service right corner of the article or click here.

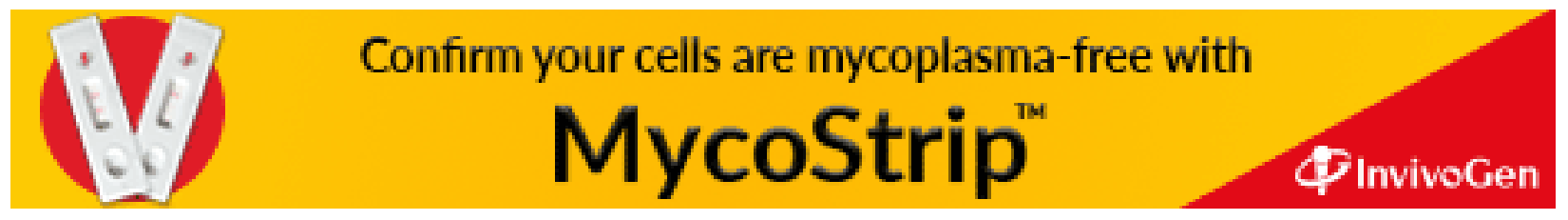

